\title{
A Fast Minutiae-Based Fingerprint Recognition System
}

\author{
Haiyun Xu, Raymond N. J. Veldhuis, Tom A. M. Kevenaar, and Ton A. H. M. Akkermans
}

\begin{abstract}
The spectral minutiae representation is a method to represent a minutiae set as a fixed-length feature vector, which is invariant to translation, and in which rotation and scaling become translations, so that they can be easily compensated for. These characteristics enable the combination of fingerprint recognition systems with template protection schemes that require as an input a fixed-length feature vector. Based on the spectral minutiae features, this paper introduces two feature reduction algorithms: the Column Principal Component Analysis and the Line Discrete Fourier Transform feature reductions, which can efficiently compress the template size with a reduction rate of $94 \%$. With reduced features, we can also achieve a fast minutiae-based matching algorithm. This paper presents the performance of the spectral minutiae fingerprint recognition system and shows a matching speed with 125000 comparisons per second on a PC with Intel Pentium D processor $2.80 \mathrm{GHz}$ and $1 \mathrm{~GB}$ of RAM. This fast operation renders our system suitable as a preselector for a large-scale fingerprint identification system, thus significantly reducing the time to perform matching, especially in systems operating at geographical level (e.g., police patrolling) or in complex critical environments (e.g., airports).
\end{abstract}

Index Terms-Biometrics, fast minutiae matching, feature reduction, fingerprint identification, template protection.

\section{INTRODUCTION}

$\mathbf{F}$ INGERPRINT recognition systems have the advantages of both ease of use and low cost. The Unisys Security Index released in December 2008 reveals that fingerprint is the most acceptable biometric technology [1]. Most fingerprint recognition systems are based on the use of a minutiae set. Minutiae are the endpoints and bifurcations of fingerprint ridges. They are known to remain unchanged over an individual's lifetime and allow a very discriminative classification of fingerprints [2].

The spectral minutiae representation presented in [3] is a method to represent a minutiae set as a fixed-length feature vector, which is invariant to translation, and in which rotation

Manuscript received January 13, 2009; revised September 18, 2009. First published November 10, 2009; current version published January 27, 2010. This work was supported by the research program Sentinels (http://www.sentinels.nl) and conducted in cooperation with priv-ID B.V. and Philips Research Laboratories.

H. Xu and R. N. J. Veldhuis are with the Department of Electrical Engineering, University of Twente, 7500 AE Enschede, The Netherlands (e-mail: h.xu@el.utwente.nl; r.n.j.veldhuis@el.utwente.nl).

T. A. M. Kevenaar is with priv-ID B.V., High Tech Campus 9, 5656 AE Eindhoven, The Netherlands (e-mail: tom.kevenaar@priv-id.com).

T. A. H. M. Akkermans is with Philips Research Laboratories, Prof. Holstlaan 4, 5656 AA Eindhoven, The Netherlands (e-mail: ton.h.akkermans@ philips.nl).

Color versions of one or more of the figures in this paper are available online at http://ieeexplore.ieee.org.

Digital Object Identifier 10.1109/JSYST.2009.2034945 and scaling become translations, so that they can be easily compensated for. These characteristics enable the combination of fingerprint recognition systems with recently developed template protection schemes based on fuzzy commitment and helper data schemes, such as [4] and [5], that require as an input a fixed-length feature vector representation of a biometric modality. ${ }^{1}$

The spectral minutiae algorithm in [3] received promising results. The spectral minutiae feature is a 32768 -dimensional real-valued feature vector. The large dimensionality of the spectral minutiae feature can cause three problems. First, the storage requirement for a spectral minutiae fingerprint system is very high. Second, the high dimensionality leads to a computational burden and the matching speed will be limited, which is not desired for fingerprint identification systems with very large databases. Third, the high dimensionality can lead to a small sample size problem [7].

In this paper, we will introduce two feature reduction methods in order to solve the above problems of the original spectral minutiae algorithm: the Column Principal Component Analysis (Column-PCA) and the Line Discrete Fourier Transform (LineDFT) feature reduction algorithms. By applying Column-PCA and Line-DFT methods to the original spectral minutiae features, we can effectively compress the spectral minutiae templates and increase the matching speed as well.

For a large Automated Fingerprint Identification System (AFIS), the recognition accuracy, matching speed and its robustness to poor image quality are normally regarded as the most critical elements of system performance. Due to the fact that minutiae sets are unordered, the correspondence between individual minutia in two minutiae sets is unknown before matching. This makes it difficult to find the geometric transformation that optimally registers (or aligns) two minutiae sets. For fingerprint identification systems with very large databases [8], in which a fast comparison algorithm is necessary, most minutiae-based matching algorithms will fail to meet the high speed requirement. Compared with other AFIS vendors, our spectral minutiae fingerprint recognition system has the speed advantage: the experiment shows that our matching speed is more than 15 times higher than that of another commercial minutiae-based fingerprint matching algorithm (we will present the details later). To satisfy the high speed requirement, some AFIS vendors first use the global fingerprint characteristics (image-based features) as the first stage matching, and then use the minutiae matcher as the second stage matching [2]. However, this requires the original fingerprint images and such

\footnotetext{
${ }^{1}$ Other template protection systems exist [6] that do not pose this fixed-length feature vector requirement.
} 
solutions cannot be integrated into the large amount of existing minutiae-based fingerprint recognition systems, in which only minutiae sets are stored as templates. The spectral minutiae representation we present in this paper only needs the minutiae templates as input, so that it can be easily integrated into any arbitrary minutiae-based fingerprint recognition system. This motivates us to consider our spectral minutiae algorithm as a preselector (or pre-filter) for a large-scale AFIS to improve the overall matching speed performance, especially in systems operating at geographical level (e.g., police patrolling) or in complex critical environments (e.g., airports). Besides the speed advantage, our algorithm can also be combined with template protection schemes, which gains more and more attention due to the substantial privacy concerns.

This paper is organized as follows. First, the background of the spectral minutiae representation is presented in Section II. Next, in Section III and Section IV, we introduce the Column-PCA and Line-DFT feature reduction algorithms. Then, Section V presents the experimental results. Finally, we draw conclusions in Section VI.

\section{SPECTRAL MinUtiae RePResentation}

The spectral minutiae representation is based on the shift, scale and rotation properties of the two-dimensional continuous Fourier transform. In [3], the concept of two representation methods are introduced: the location-based spectral minutiae representation (SML) and the orientation-based spectral minutiae representation (SMO).

\section{A. Location-Based Spectral Minutiae Representation (SML)}

When implementing the Fourier transform there are two important issues that should be considered. First, when a discrete Fourier transform is taken of an image, this results in a representation of a periodic repetition of the original image. This is undesirable because it introduces errors due to discontinuities at the image boundaries. Second, the re-mapping onto a polar-logarithmic coordinate system after using a discrete Fourier transform introduces interpolation artifacts. Therefore, we introduce an analytical representation of the input minutiae, and then use analytical expressions of a continuous Fourier transform that are evaluated on a grid in the polar-logarithmic plane. These analytical expressions are obtained as follows. Assume we have a fingerprint with $Z$ minutiae. With every minutia, a function $m_{i}(x, y)=\delta\left(x-x_{i}, y-y_{i}\right), i=1, \ldots, Z$ is associated where $\left(x_{i}, y_{i}\right)$ represents the location of the $i$ th minutia in the fingerprint image. Thus, in the spatial domain, every minutia is represented by a Dirac pulse. The Fourier transform of $m_{i}(x, y)$ is given by

$$
\mathcal{F}\left\{m_{i}(x, y)\right\}=\exp \left(-\mathrm{j}\left(\omega_{\mathrm{x}} x_{i}+\omega_{\mathrm{y}} y_{i}\right)\right)
$$

and the location-based spectral minutiae representation is defined as

$$
\mathcal{M}_{\mathrm{L}}\left(\omega_{\mathrm{x}}, \omega_{\mathrm{y}}\right)=\sum_{i=1}^{Z} \exp \left(-\mathrm{j}\left(\omega_{\mathrm{x}} x_{i}+\omega_{\mathrm{y}} y_{i}\right)\right) .
$$

In order to reduce the sensitivity to small variations in minutiae locations in the spatial domain, we use a Gaussian low-pass filter to attenuate the higher frequencies. This multiplication in the frequency domain corresponds to a convolution in the spatial domain where every minutia is now represented by a Gaussian pulse.

Following the shift property of the Fourier transform, the magnitude of $\mathcal{M}$ is taken in order to make the spectrum invariant to translation of the input and we obtain

$$
\begin{aligned}
& \left|\mathcal{M}_{\mathrm{L}}\left(\omega_{\mathrm{x}}, \omega_{\mathrm{y}} ; \sigma_{\mathrm{L}}^{2}\right)\right| \\
& \quad=\left|\exp \left(-\frac{\omega_{\mathrm{x}}^{2}+\omega_{\mathrm{y}}^{2}}{2 \sigma_{\mathrm{L}}^{-2}}\right) \sum_{i=1}^{Z} \exp \left(-\mathrm{j}\left(\omega_{\mathrm{x}} x_{i}+\omega_{\mathrm{y}} y_{i}\right)\right)\right|
\end{aligned}
$$

Equation (3) is the analytical expression for the spectrum which can directly be evaluated on a polar-logarithmic grid. The resulting representation in the polar-logarithmic domain is invariant to translation, while rotation and scaling of the input have become translations along the polar-logarithmic coordinates.

\section{B. Orientation-Based Spectral Minutiae Representation (SMO)}

The location-based spectral minutiae representation (SML) only uses the minutiae location information. However, including the minutiae orientation as well may give better discrimination. Therefore, it can be beneficial to also include the orientation information in our spectral representation. The orientation $\theta$ of a minutia can be incorporated by using the spatial derivative of $m(x, y)$ in the direction of the minutia orientation. Thus, to every minutia in a fingerprint, a function $m_{i}(x, y, \theta)$ is assigned being the derivative of $m_{i}(x, y)$ in the direction $\theta_{i}$, such that

$$
\begin{aligned}
\mathcal{F}\left\{m_{i}(x, y, \theta)\right\}=\mathrm{j}\left(\omega_{\mathrm{x}} \cos \theta_{i}\right. & \left.+\omega_{\mathrm{y}} \sin \theta_{i}\right) \\
\cdot & \exp \left(-\mathrm{j}\left(\omega_{\mathrm{x}} x_{i}+\omega_{\mathrm{y}} y_{i}\right)\right) .
\end{aligned}
$$

As with the SML algorithm, using a Gaussian filter and taking the magnitude of the spectrum yields

$$
\begin{aligned}
& \left|\mathcal{M}_{\mathrm{O}}\left(\omega_{\mathrm{x}}, \omega_{\mathrm{y}} ; \sigma_{\mathrm{O}}^{2}\right)\right|=\mid \exp \left(-\frac{\omega_{\mathrm{x}}^{2}+\omega_{\mathrm{y}}^{2}}{2 \sigma_{\mathrm{O}}^{-2}}\right) \\
& \quad \times \sum_{i=1}^{Z} \mathrm{j}\left(\omega_{\mathrm{x}} \cos \theta_{i}+\omega_{\mathrm{y}} \sin \theta_{i}\right) \cdot \exp \left(-\mathrm{j}\left(\omega_{\mathrm{x}} x_{i}+\omega_{\mathrm{y}} y_{i}\right)\right) \mid \cdot
\end{aligned}
$$

\section{Implementation}

In the previous sections we introduced analytical expressions for the spectral minutiae representations of a fingerprint. In order to obtain our final spectral representations, the continuous spectra (3) and (5) are sampled on a polar-logarithmic grid. In the radial direction $\lambda$, we use $M=128$ samples between $\lambda_{1}=0.1$ and $\lambda_{\mathrm{h}}=0.6$. In the angular direction $\beta$, we use $N=256$ samples uniformly distributed between $\beta=0$ and $\beta=\pi$. Because of the symmetry of the Fourier transform for 


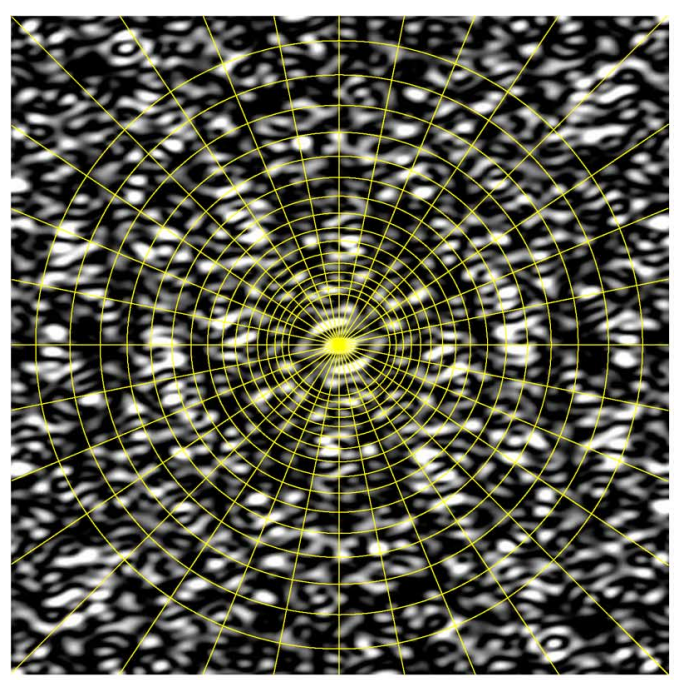

(a)

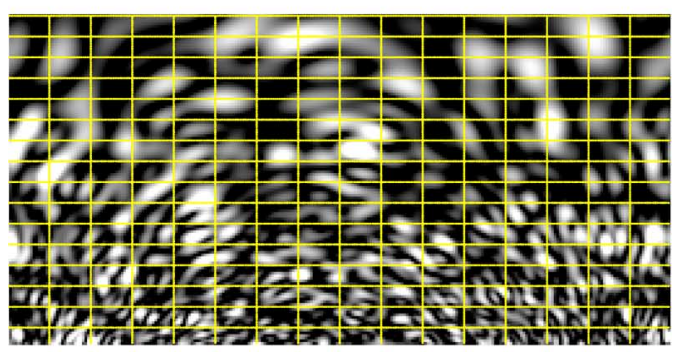

(b)

Fig. 1. Illustration of the polar-logarithmic sampling (SML spectra). (a) Fourier spectrum in a Cartesian coordinate and a polar-logarithmic sampling grid. (b) Fourier spectrum sampled on a polar-logarithmic grid.

real-valued functions, using the interval between 0 and $\pi$ is sufficient. This polar-logarithmic sampling process is illustrated in Figs. 1 and 2.

The sampled spectra (3) and (5) will be denoted by $S_{\mathrm{L}}\left(m, n ; \sigma_{\mathrm{L}}\right)$ and $S_{\mathrm{O}}\left(m, n ; \sigma_{\mathrm{O}}\right)$, respectively, with $m=1, \ldots, M, n=1, \ldots, N$. When no confusion can arise, the parameter $\sigma$ and the subscripts $\mathrm{L}$ and $\mathrm{O}$ will be omitted. For each spectrum, the horizontal axis represents the rotation angle of the spectral magnitude (from 0 to $\pi$ ); the vertical axis represents the frequency of the spectral magnitude (the frequency increases from top to bottom). It should be noted that the minutiae spectrum is periodic on the horizontal axis.

\section{Spectral Minutiae Matching}

Let $R(m, n)$ and $T(m, n)$ be the two sampled minutiae spectra respectively achieved from the reference fingerprint and the test fingerprint. Both $R(m, n)$ and $T(m, n)$ are normalized to have zero mean and unit energy. We use the two-dimensional correlation coefficient between $R$ and $T$ as a measure of their similarity.

In practice, the input fingerprint images are rotated and might be scaled (for example, depending on the sensor that is used to acquire an image). Assume that the scaling has already been compensated for on the level of the minutiae sets [9]. Then we only need to test a few rotations, which become the circular shifts in the horizontal direction. We denote $T\left(m, n-n_{c s}\right)$ as

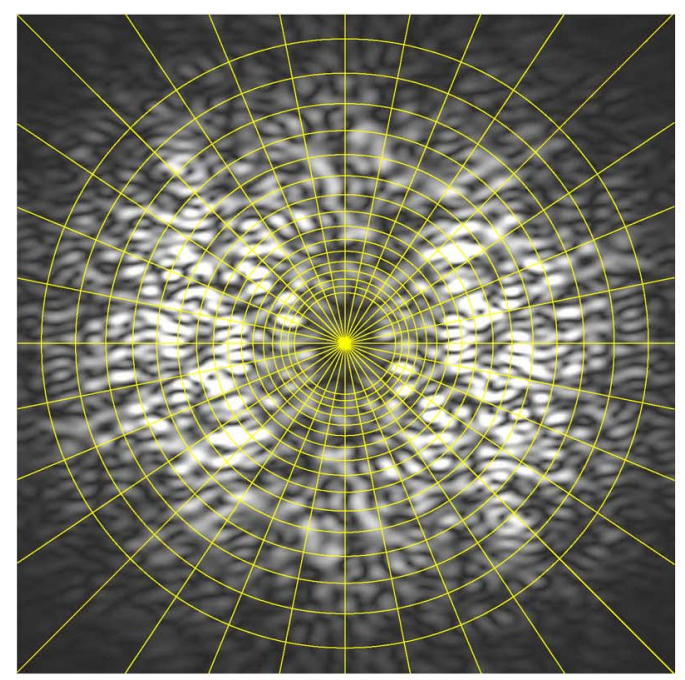

(a)

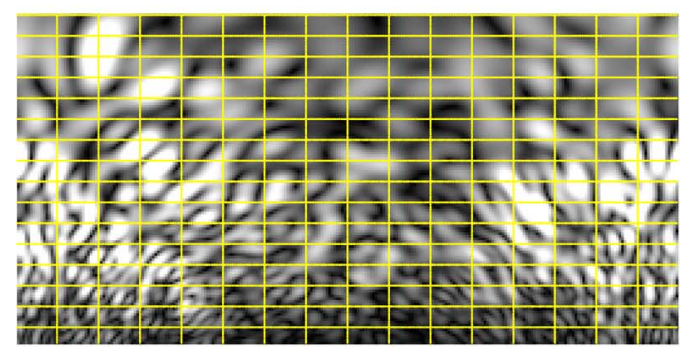

(b)

Fig. 2. Illustration of the polar-logarithmic sampling (SMO spectra). (a) Fourier spectrum in a Cartesian coordinate and a polar-logarithmic sampling grid. (b) Fourier spectrum sampled on a polar-logarithmic grid.

a circular shifted version of $T(m, n)$. We use the fast rotation searching algorithm, based on variable stepsizes that was presented in $[10]^{2}$ and choose the maximum score of the different combinations as the final matching score between $R$ and $T$

$$
\begin{array}{r}
S^{(R, T)}=\max _{n_{c s}}\left\{\frac{1}{M N} \sum_{m, n} R(m, n) T\left(m, n-n_{c s}\right)\right\}, \\
-15 \leq n_{c s} \leq 15 .
\end{array}
$$

\section{Column-PCA FeAture Reduction (CPCA)}

The spectral minutiae feature is a $d=M \times N=32,768$ real-valued feature vector. This high dimensionality can cause the small sample size problem [7]. Small sample size effects are normally encountered in applications with high dimensional features and a complex classification rule, while the number of available training samples is inadequate. A sophisticated classifier relies on assumptions about the statistics of the feature vectors that are obtained from training data. A mismatch between the actual statistics and the assumptions will lead to a loss of recognition performance. We can increase robustness against this type of mismatch by reducing the feature space dimensionality. PCA is a commonly used tool to achieve this, which at the same time decorrelates the features [11].

\footnotetext{
${ }^{2}$ In [10], a total of nine rotations are tested in a range of $-10^{\circ}$ to $+10^{\circ}$ in case of $N=256$ samples between 0 to $\pi$.
} 


\section{A. PCA Feature Reduction and Its Problem on Spectral Minutiae Representation}

In order to illustrate the problem of directly applying PCA on the features $S_{\mathrm{L}}(m, n)$ and $S_{\mathrm{O}}(m, n)$, let $\vec{x}=\left(x_{1}, \ldots, x_{D}\right)^{\mathrm{T}}$ denote the unreduced spectral minutiae feature vector, that is, a one-dimensional form of the two-dimensional spectral minutiae $S(m, n)$, with $m=1, \ldots, M, n=1, \ldots, N,(M=128$ and $N=256$ ). Thus, the dimensionality of $\vec{x}$ is $D=M \times N=$ 32,768 .

If we have $L$ samples $\vec{x}_{1}, \ldots, \vec{x}_{L}$ in the training set, we can create a $D \times L$ data matrix $\mathbf{X}$ consisting of all the samples, as $\mathbf{X}=\left[\vec{x}_{1}, \ldots, \vec{x}_{L}\right]$. PCA can be implemented by doing a singular value decomposition (SVD) on the matrix $\mathbf{X}$

$$
\mathbf{X}=\mathbf{U}_{\mathrm{X}} \mathbf{S}_{\mathrm{X}} \mathbf{V}_{\mathrm{X}}^{\mathrm{T}}
$$

with $\mathbf{U}_{\mathrm{X}}$ a $D \times L(D>L)$ orthonormal matrix spanning the column space of $\mathbf{X}, \mathbf{S}_{\mathrm{X}}$ a $L \times L$ diagonal matrix of which the (non-negative) diagonal elements are the singular values of $\mathbf{X}$ in descending order, and $\mathbf{V}_{\mathrm{X}}$ a $L \times L$ orthonormal matrix spanning the row space of $\mathbf{X}$. Let $\widetilde{\mathbf{U}}_{\mathrm{X}}$ be the submatrix of $\mathbf{U}_{\mathrm{X}}$ consisting of the first $D_{\mathrm{PCA}}\left(D_{\mathrm{PCA}}<L\right)$ columns, then we can implement PCA by

$$
\mathbf{Y}=\tilde{\mathbf{U}}_{\mathbf{X}}^{\mathrm{T}} \mathbf{X}
$$

with $\mathbf{Y}$ the $D_{\mathrm{PCA}} \times L$ data matrix with reduced dimensionality.

However, there are two problems in performing PCA to implement feature reduction on the minutiae spectra. The first is the small sample size problem [7]. In case the feature vector is an unreduced spectral minutiae representation, the dimensionality of the feature vector is $D=32,768$. A reliable PCA feature reduction requires a large number of fingerprint samples to implement the PCA training, which is difficult to acquire. The second problem is that the minutiae spectra are not rotation-invariant. As we mentioned in the previous section, the rotation of fingerprints becomes a circular shift of the minutiae spectra in the horizontal direction. For the PCA training, all the minutiae spectra must be aligned in order to get meaningful results. Then both the training and matching processes become complicated. To cope with the small sample size problem and to avoid the rotation alignment of minutiae spectra, we introduce the Column-PCA method to perform a feature reduction.

\section{B. Column-PCA: Feature Reduction Without Small Sample Size Problems}

We first look at the spectral minutiae feature $S$ in the 2-D case as we presented in Section II-C. From Figs. 1 and 2, we can see that the minutiae spectrum is periodic on the horizontal axis. Moreover, on the vertical axis, the spectra with different frequencies are correlated. Therefore, we consider to use PCA to decorrelate the spectra with different frequencies in the vertical direction. To achieve this, we regard each column of $S$ as a new feature vector $\vec{z}=\left(z_{1}, \ldots, z_{M}\right)^{\mathrm{T}}$ (we will call $\vec{z}$ a column feature vector later in this paper), then each (sampled) minutiae spectrum $S(m, n)$ consists of $N$ feature vectors $\vec{z}, S=\left(\vec{z}_{1}, \ldots, \vec{z}_{N}\right)$.

If we have $L$ samples $S_{1}, \ldots, S_{L}$ in the training set, we can create a $M \times L_{\mathrm{N}}\left(L_{\mathrm{N}}=N \times L, N=256\right)$ data matrix $\mathbf{Z}$ consists of all the samples, as $\mathbf{Z}=\left[\vec{z}_{1}, \ldots, \vec{z}_{L_{N}}\right]$. In this case, the dimensionality of the column feature vector $\vec{z}, M=128$, is $\mathrm{N}$ times smaller than the dimensionality of the spectral minutiae $D=M \times N=32,768$. At the same time, the sample size $L_{\mathrm{N}}$ is $\mathrm{N}$ times bigger than the previous sample size $L$. If we denote $r_{\mathrm{s}}$ as the rate of the sample size $l$ to the feature dimensionality $d$, $r_{\mathrm{s}}=l / d$, we can see that in case the sample number $L$ keeps the same, the $r_{\mathrm{s}}$ of using the column feature vector is $N^{2}=65,536$ times bigger than the one of using the original feature vector. Therefore, by using column feature vectors of spectral minutiae to implement PCA feature reduction, we can avoid the small sample size problem.

As we indicated in the previous section, another problem of directly using minutiae spectra to implement PCA feature reduction is that a rotation alignment of the minutiae spectra is needed, which is difficult to implement. In the spectral minutiae representation, the rotation operator commutes with column transformation. By using column feature vectors, the rotation variation becomes the samples sequence difference in the training procedure. This will not have any influence on the PCA feature reduction results. Therefore, by using column feature vectors to implement PCA feature reduction, we can cope with both the small sample size problem and avoid the rotation alignment of minutiae spectra as well. We call this method as the Column-PCA feature reduction (CPCA).

To implement CPCA, we first subtract the sample mean (column mean) from the data matrix $\mathbf{Z}$. The next step is to apply SVD on $\mathbf{Z}$

$$
\mathbf{Z}=\mathbf{U}_{\mathrm{Z}} \mathbf{S}_{\mathrm{Z}} \mathbf{V}_{\mathrm{Z}}^{\mathrm{T}}
$$

with $\mathbf{U}_{\mathrm{Z}}$ a $M \times M$ orthonormal matrix spanning the column space of $\mathbf{Z}, \mathbf{S}_{\mathrm{Z}}$ a $M \times M$ diagonal matrix of which the (non-negative) diagonal elements are the singular values of $\mathbf{Z}$ in descending order, and $\mathbf{V}_{\mathrm{Z}}$ a $L_{\mathrm{N}} \times M$ orthonormal matrix spanning the row space of $\mathbf{Z}$. Finally, we can obtain the CPCA transform matrix $\widetilde{\mathbf{U}}_{Z}$ by retaining the first $M_{\mathrm{CPCA}}\left(M_{\mathrm{CPCA}} \leq M\right)$ columns of $\mathbf{U}_{Z}$. The CPCA transform on the minutiae spectra $S(m, n)$ is written as

$$
\mathbf{S}_{\mathrm{CPCA}}=\widetilde{\mathbf{U}}_{\mathrm{Z}}^{\mathrm{T}} \mathbf{S}
$$

with $\mathbf{S}_{\mathrm{CPCA}}$ the $M_{\mathrm{CPCA}} \times N$ data matrix with reduced dimensionality. After the CPCA feature reduction, the relation of the energy retainment rate $E_{\mathrm{CPCA}}$ and $M_{\mathrm{CPCA}}$ is

$$
E_{\mathrm{CPCA}}\left(M_{\mathrm{CPCA}}\right)=\frac{\sum_{n=1}^{M_{\mathrm{CPCA}}} \mathbf{S}_{\mathrm{Z}}(n, n)}{\sum_{n=1}^{M} \mathbf{S}_{\mathrm{Z}}(n, n)}, \quad 1 \leq M_{\mathrm{CPCA}} \leq M
$$

and

$$
M_{\mathrm{CPCA}}\left(E_{\mathrm{CPCA}}\right)=\arg \min _{1 \leq \widetilde{M} \leq M}\left|\frac{\sum_{n=1}^{\widetilde{M}} \mathbf{S}_{\mathrm{Z}}(n, n)}{\sum_{n=1}^{M} \mathbf{S}_{\mathrm{Z}}(n, n)}-E_{\mathrm{CPCA}}\right| .
$$




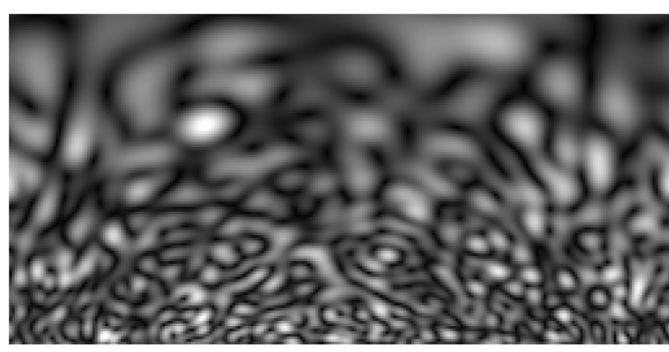

(a)

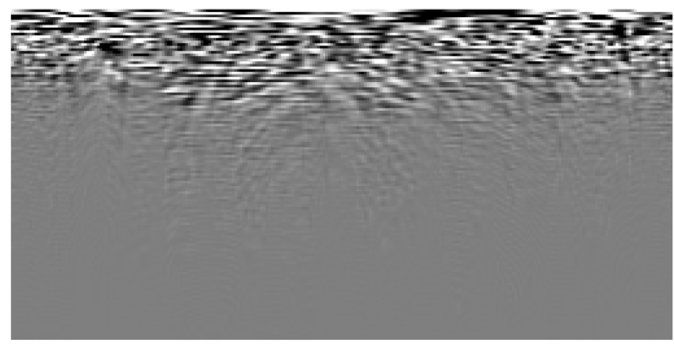

(b)

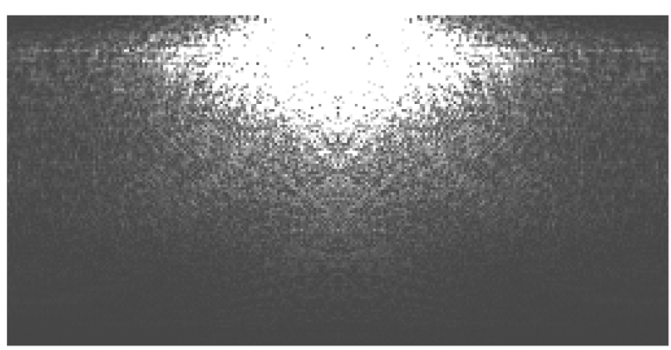

(c)

Fig. 3. Illustration of the CPCA transform and the LDFT representation. (a) Location-based spectral minutiae. (b) Minutiae spectrum after the CPCA transform. (c) Magnitude of the LDFT representation of (b).

The CPCA transform is illustrated in Fig. 3(a) and 3(b) (here, we define $\widetilde{\mathbf{U}}_{\mathrm{Z}}=\mathbf{U}_{\mathrm{Z}}$, that is, $M_{\mathrm{CPCA}}=M$ for a clear illustration). We can see that after the CPCA transform, the main energy of the original minutiae spectrum $S$ is concentrated in the top lines of $S_{\mathrm{CPCA}}$. By only retaining the top $M_{\mathrm{CPCA}}$ lines, we perform the CPCA feature reduction, with a reduction rate $R_{\mathrm{CPCA}}=\left(M-M_{\mathrm{CPCA}}\right) / M$. Because the rotation operator commutes with column transformation, the minutiae spectrum $S_{\mathrm{CPCA}}$ remains periodic on the horizontal axis after the CPCA transform.

\section{LINE-DFT FEATURE REDUCTION (LDFT)}

The CPCA feature reduction method reduces the minutiae spectrum feature $S$ in the vertical direction. In this section, we will introduce the Line-DFT feature reduction (LDFT) method, which will reduce the feature in the horizontal direction. This method is based on the fact that the minutiae spectrum $S$ is periodic on the horizontal axis. Therefore, it can be applied both independently and in combination with the CPCA feature reduction.

\section{A. Line-DFT Representation of the Minutiae Spectrum}

We denote each line of the minutiae spectrum $S$ (here $S$ can be the original minutiae spectrum or the minutiae spectrum after the CPCA feature reduction) as a line feature vector $\vec{y}=$ $\left(y_{1}, \ldots, y_{N}\right)$, thus $S=\left(\vec{y}_{1}, \ldots, \vec{y}_{M}\right)^{\mathrm{T}}$. Then we can regard each line feature vector as a periodic discrete-time signal (or sequence) with period $N$, and we denote this signal as $y_{m}(n)$, $(m=1, \ldots, M)(M=128$ for the original minutiae spectrum or $M=M_{\mathrm{CPCA}}$ for the spectrum after the CPCA feature reduction). The discrete Fourier transform [12] of $y_{m}(n)$ is given by

$$
\begin{aligned}
Y_{m}(k)= & \sum_{n=0}^{N-1} y_{m}(n) \exp \left(-\mathrm{j} \frac{2 \pi}{N} k n\right), \\
& k=0,1, \ldots, N-1, m=1, \ldots, M .
\end{aligned}
$$

Because $y_{m}(n)$ is periodic, by performing DFT (implemented as FFT) on each line $y_{m}(n)$ of the minutiae spectrum $S$, we can obtain $S_{\mathrm{LDFT}}=\left(Y_{1}(k), \ldots, Y_{M}(k)\right)^{\mathrm{T}}, S_{\mathrm{LDFT}} \in \mathbb{C}^{M}$, which is an exact representation of $S$.

The LDFT representation is illustrated in Figs. 3(b) and 3(c) (here, the LDFT representation after the CPCA feature reduction is presented). We can see that after the LDFT representation, the main energy is concentrated in the low frequency part (the middle columns). Therefore, for each line of the LDFT representation $S_{\mathrm{LDFT}}$, we only retain the Fourier components with a certain percentage of energy (for example, $80 \%$ ) in the lower frequency part. By reducing the number of Fourier components, we implement the LDFT feature reduction. For each line $m$, the relation of the energy retainment rate $E_{\mathrm{LDFT}}$ after the LDFT feature reduction and $N_{\mathrm{LDFT}}$ (which indicates that only the $N_{\text {LDFT }}$ Fourier components from the low frequency part are retained) is

$$
\begin{gathered}
E_{\mathrm{LDFT}}\left(N_{\mathrm{LDFT}} ; m\right)=\frac{\sum_{k=0}^{N_{\mathrm{LDFT}}-1}\left|Y_{m}(k)\right|^{2}}{\sum_{k=0}^{N / 2}\left|Y_{m}(k)\right|^{2}}, \\
1 \leq N_{\mathrm{LDFT}} \leq \frac{N}{2}+1
\end{gathered}
$$

and

$$
N_{\mathrm{LDFT}}\left(E_{\mathrm{LDFT}} ; m\right)=\arg \min _{1 \leq \widetilde{N} \leq \frac{N}{2}+1}\left|\frac{\sum_{k=0}^{\widetilde{N}-1}\left|Y_{m}(k)\right|^{2}}{\sum_{k=0}^{N / 2}\left|Y_{m}(k)\right|^{2}}-E_{\mathrm{LDFT}}\right| .
$$

As mentioned in Section II-D, the rotation of the fingerprint becomes the circular shift of the minutiae spectrum along the horizontal axis in the space domain. To test different fingerprint rotations (see Section II-D) after applying the LDFT representation, we will implement the shift operation in the frequency domain according to the shift property of the discrete Fourier transform. Thus, the Line-DFT transformation of $T\left(m, n-n_{c s}\right)$ in (6) becomes

$$
\begin{aligned}
T\left(m, n-n_{c s}\right)= & \left(y_{1}\left(n-n_{c s}\right), \ldots, y_{M}\left(n-n_{c s}\right)\right)^{\mathrm{T}} \\
\stackrel{\text { LDFT }}{\longrightarrow} & \exp \left(-\mathrm{j} \frac{2 \pi}{N} k n_{c s}\right) \\
& \times\left(Y_{1}(k), \ldots, Y_{M}(k)\right)^{\mathrm{T}} .
\end{aligned}
$$




\section{B. Transform of Fourier Components to a Real-Valued Feature Vector}

Consider two discrete-time, periodic signals $f_{1}[n]$ and $f_{2}[n]$, $f_{1}[n], f_{2}[n] \in \mathbb{R}^{N}$, with period $N$ ( $N$ is an even number), and their discrete Fourier transform are $F_{1}[k]$ and $F_{2}[k]$ respectively. The DFT is orthnormal, thus it preserves inner products. Therefore, because of the symmetry properties of the DFT for real-valued signals, the correlation of $f_{1}[n]$ and $f_{2}[n]$ becomes

$$
\sum_{n=0}^{N-1} f_{1}[n] f_{2}[n]=\frac{1}{N}\left(F_{1}[0] F_{2}[0]+2 \Re \sum_{k=1}^{\frac{N}{2}} F_{1}[k] F_{2}^{*}[k]\right)
$$

where $*$ denotes the complex conjugate and $\Re$ denotes the real part.

Because $F_{1}[k]$ and $F_{2}[k]$ are complex numbers, we can write them as

$$
F_{i}[k]=A_{i}[k]+\mathrm{j} B_{i}[k], \quad i=1,2
$$

with $A_{i}[k]$ the real part, and $B_{i}[k]$ the imaginary part. Then, (17) becomes

$$
\begin{aligned}
\sum_{n=0}^{N-1} f_{1}[n] f_{2}[n]= & \frac{1}{N}\left(F_{1}[0] F_{2}[0]\right. \\
& \left.+2 \sum_{k=1}^{\frac{N}{2}}\left(A_{1}[k] A_{2}[k]+B_{1}[k] B_{2}[k]\right)\right) .
\end{aligned}
$$

Therefore, we can generate two one dimensional real-valued feature vectors $\overrightarrow{v_{1}}$ and $\overrightarrow{v_{2}}$ from the Fourier components that are

$$
\begin{array}{r}
\overrightarrow{v_{i}}=\left(\frac{1}{\sqrt{N}} A_{i}[0], \sqrt{\frac{2}{N}} A_{i}[1], \ldots, \sqrt{\frac{2}{N}} A_{i}\left[\frac{N}{2}\right]\right. \\
\left.\sqrt{\frac{2}{N}} B_{i}[1], \ldots, \sqrt{\frac{2}{N}} B_{i}\left[\frac{N}{2}\right]\right), \quad i=1,2 .
\end{array}
$$

The correlation of $\overrightarrow{v_{1}}$ and $\overrightarrow{v_{2}}$ is exactly the same as the correlation of the real-valued signals $f_{1}[n]$ and $f_{2}[n]$. Therefore, by generating the new feature vectors as $\overrightarrow{v_{1}}$ and $\overrightarrow{v_{2}}$, we can continue using the correlation-based spectral minutiae matching algorithm. Moreover, by performing the correlation of $\overrightarrow{v_{1}}$ and $\overrightarrow{v_{2}}$, instead of implementing the complex number multiplications as in (17), we can save about half of the real multiplications.

In case the LDFT feature reduction, the reduced feature vectors $\overrightarrow{v_{1}}$ and $\overrightarrow{v_{2}}$ become

$$
\begin{aligned}
\overrightarrow{v_{i}}= & \left(\frac{1}{\sqrt{N}} A_{i}[0], \sqrt{\frac{2}{N}} A_{i}[1], \ldots, \sqrt{\frac{2}{N}} A_{i}\left[N_{\mathrm{LDFT}}-1\right],\right. \\
& \left.\sqrt{\frac{2}{N}} B_{i}[1], \ldots, \sqrt{\frac{2}{N}} B_{i}\left[N_{\mathrm{LDFT}}-1\right]\right), \quad i=1,2 .
\end{aligned}
$$

For the matching algorithm presented in Section II-D, we denote $v_{r}$ and $v_{t, n_{c s}}$ as the reduced features of $R(m, n)$ and $T\left(m, n-n_{c s}\right)$, respectively, then (6) becomes

$$
S^{(R, T)}=\max _{n_{c s}}\left\{\frac{1}{M N} \sum v_{r} v_{t, n_{c s}}\right\}, \quad-15 \leq n_{c s} \leq 15 .
$$

\section{EXPERIMENTS}

\section{A. Measurements}

We test the spectral minutiae representation in a verification setting. The matching performance of a fingerprint verification system can be evaluated by means of several measures. Commonly used are the false acceptance rate (FAR), the false rejection rate (FRR), and the equal error rate (EER). In this paper, we use FAR, EER and the genuine accept rate (GAR), GAR = $1-\mathrm{FRR}$, as performance indicators of our scheme.

\section{B. Experimental Settings}

The proposed algorithms have been evaluated on MCYT [13] and FVC2002-DB2 [14] fingerprint databases. The fingerprint data that we used from MCYT are obtained from 145 individuals (person ID from 0000 to 0144 and finger ID 0) and each individual contributes 12 samples. We use samples from person ID 0100 to 0144 for the CPCA and LDFT training (total 540 fingerprints) and samples from person ID 0000 to 0099 for test (total 1200 fingerprints). We also tested our algorithms on the FVC2002-DB2 because it is a public-domain fingerprint database. Compared with MCYT, the fingerprints in FVC2002 have lower quality and bigger displacements. For the FVC database, we apply the same experimental protocol as in the FVC competition: the samples from finger ID 101 to 110 for the CPCA and LDFT training (total 40 fingerprints) and samples from person ID 1 to 100 for test (total 400 fingerprints). ${ }^{3}$ The minutiae sets were obtained by the VeriFinger minutiae extractor [15]. ${ }^{4}$

We test our algorithm in a verification setting. For matching genuine pairs, we used all the possible combinations. For matching imposter pairs, we chose the first sample from each identity. We will further follow the same parameter setting in $[3] .5^{5}$

\section{Results Without CPCA and LDFT Feature Reductions}

For a comparison with the results after the CPCA and the LDFT feature reductions, we first tested our algorithm without feature reductions. The results are shown in Table I and the ROC curves are shown in Figs. 4(a) and 5(a). From the results, we can see that the MCYT database received much better results than

\footnotetext{
${ }^{3}$ We propose to use our algorithm in a high security scenario. In FVC2002 databases, samples $3,4,5$, and 6 were obtained by requesting the users to provide fingerprints with exaggerated displacement and rotation. In a high security scenario where the user is aware that cooperation is crucial for security reasons, he will be cooperative. Therefore, only samples $1,2,7$, and 8 are chosen. To deal with the large rotations, an absolute pre-alignment based on core and its direction can be applied.

${ }^{4}$ VeriFinger Extractor Version 5.0.2.0 is used.

${ }^{5}$ We will only present the sum-rule fusion results of SML and SMO in this paper. We also use the singular points to assist the verification, following the procedure in [3].
} 
TABLE I

RESULTS WITHOUT CPCA AND LDFT FEATURE REDUCTIONS

\begin{tabular}{|l|c|c|c|c|}
\hline \multirow{2}{*}{ Databases } & \multirow{2}{*}{ EER } & \multicolumn{3}{|c|}{ GAR } \\
\cline { 3 - 5 } & & FAR $=1 \%$ & FAR $=0.1 \%$ & FAR $=0 \%$ \\
\hline MCYT & $0.30 \%$ & $99.8 \%$ & $99.7 \%$ & $99.1 \%$ \\
\hline FVC2002-DB2 & $3.86 \%$ & $95.5 \%$ & $92.7 \%$ & $89.7 \%$ \\
\hline
\end{tabular}

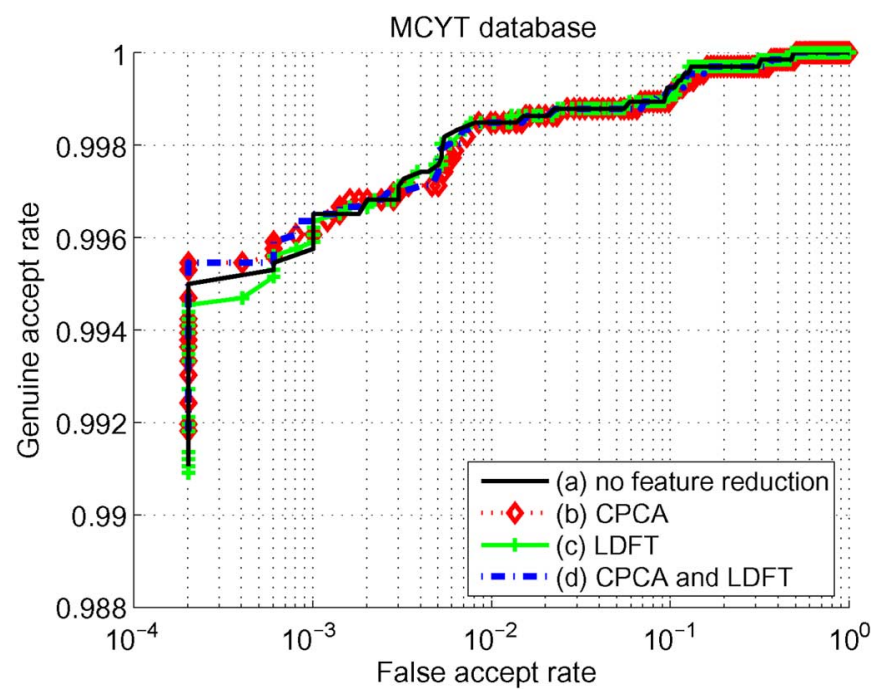

Fig. 4. ROC curves (MCYT database).

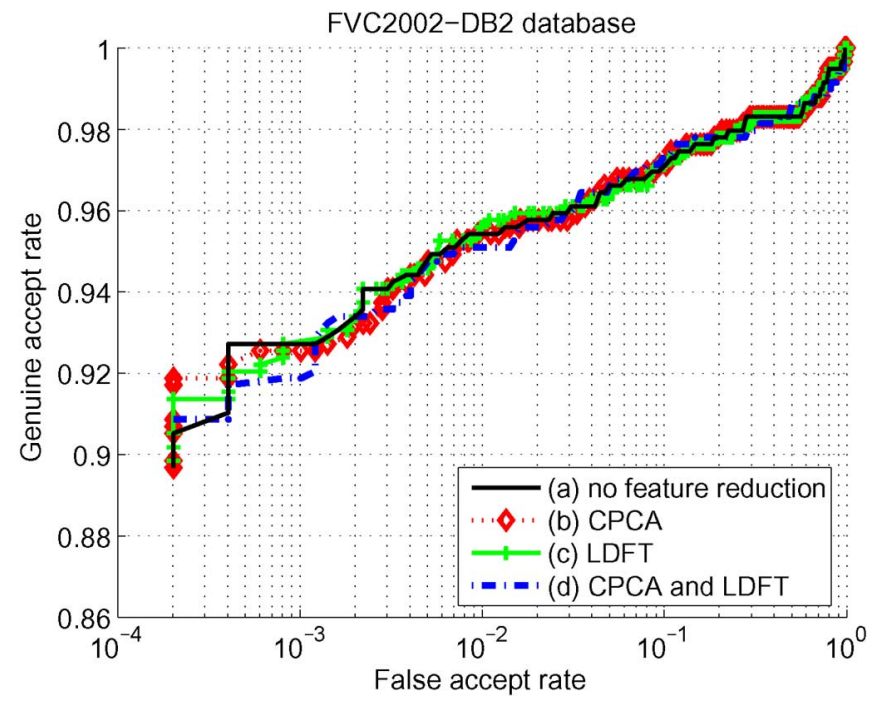

Fig. 5. ROC curves (FVC2002-DB2 database).

the FVC database. This shows that our algorithms are sensitive to the minutiae quality and fingerprint quality.

\section{Results After CPCA and LDFT Feature Reductions}

In case of using SML and SMO fusion, the spectral minutiae representation results in a 65536 real-valued feature vector. For fingerprint identification systems with very large databases, using the spectral minutiae representation requires a big template storage space and its matching speed is also limited. Therefore, applying the proposed CPCA and LDFT feature reduction algorithms is needed. To evaluate the two feature reduction methods, we tested them in three cases: 1) only applying the
TABLE II

PARAMETERS OF THE CPCA FEATURE Reduction

\begin{tabular}{|l|c|c|c|c|c|c|}
\hline \multirow{2}{*}{} & \multicolumn{3}{|c|}{ MCYT } & \multicolumn{3}{c|}{ FVC2002-DB2 } \\
\cline { 2 - 7 } & SML & SMO & Fusion & SML & SMO & Fusion \\
\hline$E_{\text {CPCA }}$ & $85 \%$ & $90 \%$ & $87.5 \%$ & $85 \%$ & $90 \%$ & $87.5 \%$ \\
\hline Reduction & $76.6 \%$ & $78.1 \%$ & $77.3 \%$ & $67.2 \%$ & $76.6 \%$ & $71.9 \%$ \\
\hline
\end{tabular}

TABLE III

Results After the CPCA Feature Reduction

\begin{tabular}{|l|c|c|c|c|}
\hline \multirow{2}{*}{ Databases } & \multirow{2}{*}{ EER } & \multicolumn{3}{|c|}{ GAR } \\
\cline { 3 - 5 } & & FAR $=1 \%$ & FAR $=0.1 \%$ & FAR $=0 \%$ \\
\hline MCYT & $0.30 \%$ & $99.8 \%$ & $99.6 \%$ & $99.2 \%$ \\
\hline FVC2002-DB2 & $3.72 \%$ & $95.4 \%$ & $92.6 \%$ & $89.7 \%$ \\
\hline
\end{tabular}

TABLE IV

PARAMETERS OF THE LDFT FEATURE REDUCTION

\begin{tabular}{|l|c|c|c|c|c|c|}
\hline & \multicolumn{3}{|c|}{ MCYT } & \multicolumn{3}{c|}{ FVC2002-DB2 } \\
\cline { 2 - 7 } & SML & SMO & Fusion & SML & SMO & Fusion \\
\hline$E_{\text {LDFT }}$ & $97 \%$ & $99.5 \%$ & $98.3 \%$ & $97 \%$ & $99.8 \%$ & $98.4 \%$ \\
\hline Reduction & $81.4 \%$ & $88.5 \%$ & $84.9 \%$ & $77.5 \%$ & $89.0 \%$ & $83.3 \%$ \\
\hline
\end{tabular}

CPCA feature reduction; 2) only applying the LDFT feature reduction; and 3) applying both the CPCA and the LDFT feature reductions.

From our experiments, we noticed that the selection of the energy retainment rates $E_{\mathrm{CPCA}}$ and $E_{\mathrm{LDFT}}$ are essential for a high performance. When $E_{\mathrm{CPCA}}$ and $E_{\mathrm{LDFT}}$ are chosen, we can calculate $M_{\mathrm{CPCA}}$ and $N_{\mathrm{LDFT} m}$ using the fingerprints in the training sets, according to (12) and (15).

1) Only Applying the CPCA Feature Reduction: When only applying the CPCA feature reduction, the energy retainment rates $E_{\mathrm{CPCA}}$ and the feature reduction rates for the two databases are shown in Table II. The results we achieved are shown in Table III and the ROC curves are shown in Figs. 4(b) and 5(b). From Figs. 4(b) and 5(b), we can see that the CPCA feature reduction does not degrade the recognition performance of the system. At the same time, we reach a feature reduction rate of more than $70 \%$ (the feature length is about four times smaller). In the FVC2002-DB2 case, we only used 40 fingerprints for the training and we still performed an effective feature reduction. This illustrates that the CPCA feature reduction does not suffer from the small sample size problem.

2) Only Applying the LDFT Feature Reduction: When only applying the LDFT feature reduction, the energy retainment rates $E_{\mathrm{LDFT}}$ and the feature reduction rates for the two databases are shown in Table IV. From Table IV, we can see that we achieved a higher reduction rate for SMO, at the same time the energy retainment is also higher. The reason is that for SMO, we used a Gaussian kernel to attenuate the higher frequencies. The LDFT feature reduction can achieve a higher reduction rate in case the minutiae spectra are with lower frequencies.

The results we achieved after the LDFT feature reduction are shown in Table V and the ROC curves are shown in Figs. 4(c) and 5(c). From Figs. 4(c) and 5(c), we can see that the LDFT feature reduction does not degrade the recognition performance of the system. At the same time, we reach a feature reduction rate 
TABLE V

RESULTS AFTER THE LDFT FEATURE REDUCTION

\begin{tabular}{|l|c|c|c|c|}
\hline \multirow{2}{*}{ Databases } & \multirow{2}{*}{ EER } & \multicolumn{3}{|c|}{ GAR } \\
\cline { 3 - 5 } & & FAR $=1 \%$ & FAR $=0.1 \%$ & FAR $=0 \%$ \\
\hline MCYT & $0.29 \%$ & $99.8 \%$ & $99.7 \%$ & $99.1 \%$ \\
\hline FVC2002-DB2 & $3.72 \%$ & $95.6 \%$ & $92.7 \%$ & $89.7 \%$ \\
\hline
\end{tabular}

TABLE VI

PARAMETERS OF THE CPCA AND THE LDFT FEATURE REDUCTIONS

\begin{tabular}{|l|c|c|c|c|c|c|}
\hline \multirow{2}{*}{ Methods } & \multicolumn{3}{|c|}{ MCYT } & \multicolumn{3}{c|}{ FVC2002-DB2 } \\
\cline { 2 - 7 } & SML & SMO & Fusion & SML & SMO & Fusion \\
\hline \hline$E_{\text {CPCA }}$ & $83 \%$ & $90 \%$ & $86.5 \%$ & $75 \%$ & $92 \%$ & $83.5 \%$ \\
\hline Reduction & $78.1 \%$ & $78.1 \%$ & $78.1 \%$ & $75 \%$ & $75 \%$ & $75 \%$ \\
\hline \hline$E_{\text {LDFT }}$ & $99 \%$ & $99 \%$ & $99 \%$ & $97 \%$ & $98 \%$ & $97.5 \%$ \\
\hline Reduction & $69.5 \%$ & $74.2 \%$ & $72.0 \%$ & $68.1 \%$ & $72.5 \%$ & $70.4 \%$ \\
\hline \hline$E_{\text {Total }}$ & $82.2 \%$ & $89.1 \%$ & $85.6 \%$ & $72.8 \%$ & $90.2 \%$ & $81.5 \%$ \\
\hline Reduction & $92.9 \%$ & $94.0 \%$ & $93.5 \%$ & $92.0 \%$ & $93.1 \%$ & $92.6 \%$ \\
\hline
\end{tabular}

TABLE VII

RESULTS AFTER THE CPCA AND THE LDFT FEATURE REDUCTIONS

\begin{tabular}{|l|c|c|c|c|}
\hline \multirow{2}{*}{ Databases } & \multirow{2}{*}{ EER } & \multicolumn{3}{|c|}{ GAR } \\
\cline { 3 - 5 } & & FAR $=1 \%$ & FAR $=0.1 \%$ & FAR $=0 \%$ \\
\hline MCYT & $0.30 \%$ & $99.8 \%$ & $99.7 \%$ & $99.2 \%$ \\
\hline FVC2002-DB2 & $3.52 \%$ & $95.1 \%$ & $92.0 \%$ & $90.7 \%$ \\
\hline
\end{tabular}

of about $84 \%$ (the feature length is more than 6 times smaller). The same as the CPCA feature reduction, the LDFT feature reduction also does not suffer from the small sample size problem.

3) Applying Both the CPCA and the LDFT Feature Reductions: After testing the CPCA and the LDFT feature reductions separately, we tested the combination of the two methods. We applied the LDFT feature reduction after the CPCA feature reduction. The final energy retainment rates $E_{\mathrm{CPCA}}$ and $E_{\mathrm{LDFT}}$, and the feature reduction rates for the two databases are shown in Table VI. The results we achieved are shown in Table VII and the ROC curves are shown in Figs. 4(d) and 5(d). From Figs. 4(d) and 5(d), we can see that after applying the CPCA and the LDFT feature reductions, the recognition performance is not degraded. We finally reach a feature reduction rate of about $92 \%-94 \%$ (the feature length is more than $13-15$ times smaller).

Comparing the three different feature reduction cases [the ROC curves in Figs. 4(b)-(d) and 5(b)-(d)], we can see that all these three cases show comparable recognition performances, while the combination of CPCA and LDFT received the biggest feature reduction rates.

For fingerprint identification systems with very large databases, the matching speed is crucial. In case the feature length is $d$ and $n_{\mathrm{r}}$ rotation possibilities are tested (in our experiments $n_{\mathrm{r}}=9$ ), we need to implement $n_{\mathrm{r}} d$ real multiplications. Therefore, applying the feature reductions to decrease $d$ will improve the speed performance of our scheme. We tested the matching speed for the fusion case of SML and SMO before and after the CPCA and the LDFT feature reductions (using the MCYT database). Without feature reductions, we can implement 8,000 comparisons per second using optimized C language programming on a $\mathrm{PC}$ with Intel Pentium $\mathrm{D}$ processor $2.80 \mathrm{GHz}$ and $1 \mathrm{~GB}$ of RAM. After applying CPCA and LDFT,
TABLE VIII

RESULTS COMPARISON ON FVC2002-DB2

\begin{tabular}{|l|c|c|c|c|}
\hline \multirow{2}{*}{ Methods } & \multirow{2}{*}{ EER } & \multicolumn{3}{|c|}{ GAR } \\
\cline { 3 - 5 } & & FAR $=1 \%$ & FAR $=0.1 \%$ & FAR $=0 \%$ \\
\hline Our method & $3.52 \%$ & $95.1 \%$ & $92.0 \%$ & $90.7 \%$ \\
\hline VeriFinger & $1.0 \%$ & $99 \%$ & $98.8 \%$ & $98.6 \%$ \\
\hline Fuzzy Vault & - & - & $91 \%$ & $86 \%$ \\
\hline
\end{tabular}

we can implement 125000 comparisons (the speed is more than 15 times higher) under the same setting.

\section{E. Comparison}

We compared our results with other well-known minutiae matchers on the FVC2002-DB2 database: VeriFinger ${ }^{6}$ and Fuzzy Vault according to the protocol in [6]. The results are shown in Table VIII. We notice that the commercial minutiae matcher VeriFinger received much better results than ours. One reason is that the VeriFinger matcher uses some fingerprint features that are not defined in the ISO minutiae template [9]. Another reason is that, with our global representation, we cannot perform minutiae pair searching, which is a crucial step for the minutiae-based matching. These two reasons may cause the degradation of our algorithm compared with VeriFinger.

We also compared the performance of our method with a minutiae-based fingerprint recognition system combined with a template protection scheme based on fuzzy vault, which is presented in [6]. The reason of this comparison is that in [6] an alignment between a fingerprint pair using minutiae information is also not possible. It should be noted that [6] includes a template protection scheme, whereas our system does not. Moreover, because [6] implemented an alignment using high curvature points, this caused a $2 \%$ failure to capture rate (FTCR), while our method does not suffer from this.

Regarding the speed performance, ${ }^{7}$ using the spectral minutiae representation after the CPCA and the LDFT feature reductions, we can implement 125000 comparisons per second. We also tested the VeriFinger matcher using the same PC setting and the matching speed is 8,000 comparisons per second. Our matching speed advantage is due to the fact that our algorithm uses a fixed-length feature vector and avoids fingerprint alignment. After applying CPCA and LDFT, the feature length is greatly reduced, which leads to a promising matching speed.

In case of fingerprint identification systems with very large databases, we might combine good identification performance and speed by using the spectral minutiae as a preselector, that finds a number of best matches and then use a standard minutiae comparison for a good accuracy. As a preselector, the recognition performance in the area of high GAR is important. We show the performance of the spectral minutiae in this area in Table IX. From Table IX, we can see that in case of good quality fingerprints (MCYT), we can use the spectral minutiae algorithm as a preselector to speed up the minutiae-based matching algorithm.

\footnotetext{
${ }^{6}$ VeriFinger Extractor Version 5.0.2.0 and VeriFinger Matcher version 5.0.2.1 are used.

${ }^{7}$ For fingerprint identification systems with large databases, only matching time is crucial. For the enrollment speed, because our algorithm only uses onesample enrollment, our enrollment time is comparable to the one from VeriFinger.
} 
TABLE IX

PERFormances AFTER CPCA AND LDFT FOR High GAR

\begin{tabular}{|l|c|c|c|}
\hline \multirow{2}{*}{ Databases } & \multicolumn{3}{|c|}{ FAR } \\
\cline { 2 - 4 } & GAR $=100 \%$ & GAR $=99.9 \%$ & GAR $=99 \%$ \\
\hline MCYT & $49.4 \%$ & $6.7 \%$ & $0 \%$ \\
\hline FVC2002-DB2 & $90.0 \%$ & $88.5 \%$ & $45.5 \%$ \\
\hline
\end{tabular}

However, the spectral minutiae algorithm is not robust to the low quality fingerprints. The fingerprint outliers will degrade the recognition accuracy, which limits the application of the spectral minutiae algorithm.

\section{CONCLUSIONS}

The spectral minutiae representation is a novel method to represent a minutiae set as a fixed-length feature vector, which is invariant to translation, and in which rotation and scaling become translations, so that they can be easily compensated for. Based on the spectral minutiae feature, this paper introduces two feature reduction methods: the Column-PCA and the Line-DFT feature reduction algorithms. The experiments show that these methods effectively decrease the spectral minutiae feature dimensionality with a reduction rate of $94 \%$, while at the same time, the recognition performance of the fingerprint system is not degraded. The proposed spectral minutiae fingerprint recognition system also shows a promising matching speed with 125000 comparisons per second. This algorithm overcomes the speed disadvantage of most minutiae-based algorithms and enables the application of a minutiae-based fingerprint identification system with a large database.

The spectral minutiae representation also enables the combination of fingerprint recognition systems and template protection schemes. In order to be able to apply the spectral minutiae representation with a template protection scheme, for example based on a fuzzy extractor [16], the next step would be to extract bits that are stable for the genuine user and completely random for an arbitrary user. For example, we can apply 2-D Gabor filters for bit extraction, which has been used in iris codes [17]. Another possibility is to first apply additional dimensionality reduction by a combination of PCA and LDA and then apply single bit extraction according to the reliable component scheme or multibit extraction [18].

In this paper, we presents the experimental results using two fingerprint databases: the MCYT and the FVC2002-DB2 databases. The MCYT database gives much better results than the FVC database. This shows that our algorithms are sensitive to the minutiae quality as well as the fingerprint quality. To cope with the low quality fingerprints and minutiae errors are topics of our further research.

\section{REFERENCES}

[1] Spotlight on Biometrics [Online]. Available: http://www.unisyssecurityindex.com//

[2] D. Maltoni, D. Maio, A. Jain, and S. Prabhakar, Handbook of Fingerprint Recognition. New York: Springer, 2003.

[3] H. Xu, R. Veldhuis, A. Bazen, T. Kevenaar, T. Akkermans, and B. Gokberk, "Fingerprint verification using spectral minutiae representations," IEEE Trans. Inf. Forensics Security, vol. 4, no. 3, pp. 397-409, Sep. 2009.
[4] P. Tuyls, A. Akkermans, T. Kevenaar, G. Schrijen, A. Bazen, and R. Veldhuis, "Practical biometric authentication with template protection," in Proc. AVBPA, 2005 , pp. 436-446.

[5] A. Juels, "Fuzzy commitment," in Security With Noisy Data-On Private Biometrics, Secure Key Storage and Anti-Counterfeiting, P. Tuyls, B. Skoric, and T. Kevenaar, Eds. : : Springer, 2007, ch. 3, pp. 45-56.

[6] K. Nandakumar, A. Jain, and S. Pankanti, "Fingerprint-based fuzzy vault: Implementation and performance," IEEE Trans. Inf. Forensics Security, vol. 2, no. 4, pp. 744-757, Dec. 2007.

[7] S. Raudys and A. Jain, Small Sample Size Effects in Statistical Pattern Recognition: Recommendations for Practitioners vol. 13, no. 3, pp. 252-264, Mar. 1991.

[8] U.S. Visitor and Immigrant Status Indicator Technology Program (USVISIT) [Online]. Available: http://www.dhs.gov/us-visit

[9] ISO/IEC 19794-2, Information Technology-Biometric Data Interchange Format_-Part 2: Finger Minutiae Data 2005.

[10] H. Xu, R. N. J. Veldhuis, T. A. M. Kevenaar, A. H. M. Akkermans, and A. M. Bazen, "Spectral minutiae: A fixed-length representation of a minutiae set," in Proc. IEEE Computer Society Conference on Computer Vision and Pattern Recognition-Workshop on Biometrics, Anchorage, AK, 2008.

[11] R. Duda, P. Hart, and D. Stork, Pattern Classification, 2nd ed. New York: Wiley-Interscience, Oct. 2000.

[12] A. V. Oppenheim, A. S. Willsky, and S. H. Nawab, Signals \& Systems, 2nd ed. Upper Saddle River, NJ: Prentice-Hall, 1996.

[13] J. Ortega-Garcła et al., "MCYT baseline corpus: A bimodal biometric database," IEE Proc. Vision, Image and Signal Processing, vol. 150, no. 6, pp. 395-401, 2003.

[14] D. Maio, D. Maltoni, R. Cappelli, J. Wayman, and A. Jain, FVC2002: Second Fingerprint Verification Competition vol. 3, pp. 811-814, Aug. 2002.

[15] VeriFinger SDK [Online]. Available: http://www.neurotechnologija.com/

[16] Y. Dodis, L. Reyzin, and A. Smith, "Fuzzy extractors," in Security With Noisy Data-On Private Biometrics, Secure Key Storage and AntiCounterfeiting, P. Tuyls, B. Skoric, and T. Kevenaar, Eds. New York: Springer, 2007, ch. 3, pp. 45-56.

[17] J. Daugman, "How iris recognition works," IEEE Trans. Circuits Syst. Video Technol., vol. 14, no. 1, pp. 21-30, Jan. 2004.

[18] C. Chen, R. Veldhuis, A. Akkermans, and T. Kevenaar, "Biometric binary string generation with detection rate optimized bit allocation," in Proc. IEEE Computer Society Conf. Computer Vision and Pattern Recognition-Workshop on Biometrics, Anchorage, AK, 2008.

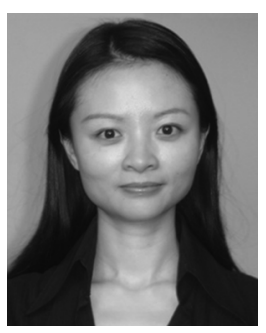

Haiyun Xu received the B.E. and M.E. degrees in electrical engineering from Beijing University of Technology, Beijing, China, in 2000 and 2003, respectively. She is currently pursuing the $\mathrm{Ph} . \mathrm{D}$. degree with the Department of Electrical Engineering, University of Twente, Enshede, The Netherlands.

From 2003 to 2005, she was with Sony and Siemens Ltd. China, respectively. She is currently working on two research projects: Protection of Biometric Templates (ProBiTe) and TrUsted Revocable Biometric IdeNtitiEs (TURBINE). Her research focuses on the integration of biometric identification in security systems. Her research interests include biometrics, pattern recognition, signal processing, and security.

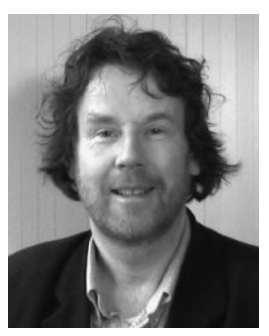

Raymond N. J. Veldhuis received the M.Sc. degree from the University of Twente, Enschede, The Netherlands in 1981 and the Ph.D. degree from Radboud University Nijmegen, Nijmegen, The Netherlands, in 1988.

From 1982 to 1992, he was with Philips Research Laboratories, Eindhoven, The Netherlands, working in various areas of digital signal processing, including signal restoration and source coding. From 1992 to 2001, he was with the Institute of Perception Research (IPO) Eindhoven, working in speech signal processing and synthesis. He is now an Associate Professor at the University of Twente, working in biometrics and pattern recognition. He has over 120 publications in international conferences and journals. 


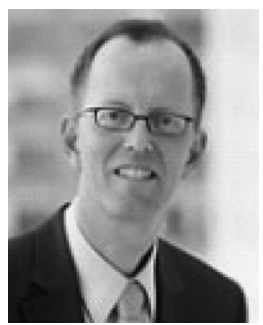

Tom A. M. Kevenaar received the Ph.D. degree from the Technical University of Eindhoven, Eindhoven, The Netherlands, in 1993.

He worked on several subjects in the field of design automation for analo and RF circuits, first at the University of Eindhoven and Hitachi Central Research Laboratory, Tokyo, Japan, and later with Philips Research. In 2001, he became involved in privacy-enhancing technologies for biometric applications. In 2008, he co-founded a start-up company, which develops and sells products to protect the privacy of biometric information. He has over 40 publications and is co-editor of the book Security with Noisy Data (Berlin, Germany: Springer, 2007).

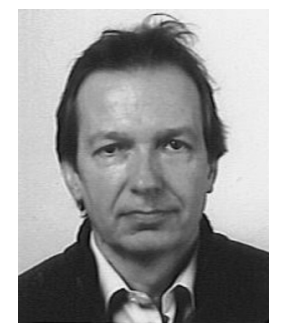

Ton A. H. M. Akkermans was born in 1959 in Breda, The Netherlands. He received the M.Sc. degree in theoretical electrical engineering from Eindhoven University of Technology, Eindhoven, The Netherlands, in 1987.

In 1982, he joined Philips Research Laboratories, Eindhoven, The Netherlands, where he worked on optical systems. In 1987, he became a Senior System Architect at Philips Optical Storage, responsible for mechatronical architectures of DVD systems. In 2000, he joined Philips Research as a Principal Scientist, where he developed architectures for copy control, DRM key-management systems, watermarking, and biometrics. Currently, he is working on biometric and safety systems. He has published numerous papers and is the holder of several patents. 